\title{
Biplots of Compositional Data
}

\author{
John Aitchison ${ }^{1}$ \\ and \\ Michael Greenacre ${ }^{2}$
}

Keywords: Logratio transformation; Principal component analysis; Relative variation biplot; Singular value decomposition; Subcomposition.

Journal of Economic Literature Classification: C19, C88.

\footnotetext{
${ }^{1}$ Department of Statistics, University of Glasgow, Glasgow, U.K. E-mail: John.Aitchison@btinternet.com

${ }^{2}$ Department of Economics and Business, Universitat Pompeu Fabra, Ramon Trias Fargas 25-27, 08005 Barcelona, Spain. E-mail: michael@upf.es
} 


\section{Summary}

The singular value decomposition and its interpretation as a linear biplot has proved to be a powerful tool for analysing many forms of multivariate data. Here we adapt biplot methodology to the specific case of compositional data consisting of positive vectors each of which is constrained to have unit sum. These relative variation biplots have properties relating to special features of compositional data: the study of ratios, subcompositions and models of compositional relationships. The methodology is demonstrated on a data set consisting of six-part colour compositions in 22 abstract paintings, showing how the singular value decomposition can achieve an accurate biplot of the colour ratios and how possible models interrelating the colours can be diagnosed. 


\section{Introduction}

Compositional data (Aitchison, 1986) consist of vectors of positive values summing to a unit, or in general to some fixed constant for all vectors. Such data arise in many disciplines, for example, in geology as major oxide compositions of rocks, in sociology and psychology as time budgets, that is parts of a time period allocated to various activities, in politics as proportions of the electorate voting for different political parties, and in genetics as frequencies of genetic groups within populations. The biplot (Gabriel, 1971) is a method which has been regularly applied to visualize the rows and columns of many different kinds of data matrices. In almost all cases, the original data values require transforming in order to depict correctly the structures that are appropriate to the particular nature of the data. Compositional data are also special in this respect and careful consideration of the relationships between parts of a composition is required before we embark on applying biplot methodology to such data.

As an example of compositional data we consider the data of Table 1, showing six-part colour compositions in 22 paintings by an amateur abstract artist. In each painting the artist uses black and white, the primary colours blue, red and yellow, and one further colour, labelled "other", which varies from painting to painting. The data are the proportions of surface area occupied by the six colours. For example, the first painting has $12.5 \%$ of the area in black, $24.3 \%$ in white, and so on. There is considerable variation from painting to painting in these colour compositions and the challenge is to describe the patterns of variability appropriately in simple terms while maintaining the unit sum constraint. An important aspect is how to treat so-called subcompositions, for example if the analysis is restricted to the three primary colours then the results should be consistent with those obtained from the full composition.

\section{Insert Table 1 about here}

In Section 2 we define the linear biplot and briefly summarize some known results which will be relevant to its application to compositional data. In Section 3 we discuss 
what makes compositional data different from interval- or ratio-scaled measurements and how to transform such data in order to perform what we shall call a relative variation biplot. In Section 4 we apply the relative variation biplot to the colour composition data and discuss issues of interpretation and modelling. Section 5 concludes with a discussion and comparison with methods such as regular principal component analysis and correspondence analysis.

\section{Biplots}

A biplot is a graphical display of the rows and columns of a rectangular $n \times p$ data matrix $\mathbf{X}$, where the rows are often individuals or other sample units, and the columns are variables. In almost all applications, biplot analysis starts with performing some transformation on $\mathbf{X}$, depending on the nature of the data, to obtain a transformed matrix $\mathbf{Z}$ which is the one that is actually displayed. Examples of transformations are centring with respect to variable means, normalization of variables, square root and logarithmic transforms.

Suppose that the transformed data matrix $\mathbf{Z}$ has rank $r$. Then $\mathbf{Z}$ can be factorized as the product:

$$
\mathbf{Z}=\mathbf{F G}^{\top}
$$

where $\mathbf{F}$ is $n \times r$ and $\mathbf{G} p \times r$. The rows of $\mathbf{F}$ and the rows of $\mathbf{G}$ provide the coordinates of $n$ points for the rows and $p$ points for the columns in an $r$-dimensional Euclidean space, called the full space since it has as many dimensions as the rank of $\mathbf{Z}$. This joint plot of the two sets of points can be referred to as the exact biplot in the full space. There are an infinite number of ways to choose $\mathbf{F}$ and $\mathbf{G}$, and certain choices favour the display of the rows, others the display of the columns. But for any particular choice the biplot in $r$ dimensions has the property that the scalar product between the $i$-th row point and $j$-th column point with respect to the origin is equal to the $(i, j)$-th element $z_{i j}$ of $\mathbf{Z}$.

We are mainly interested in low-dimensional biplots of $\mathbf{Z}$, especially in two dimensions, and these can be conveniently achieved using the singular value decomposition (SVD) of 
Z:

$$
\mathbf{Z}=\mathbf{U} \Gamma \mathbf{V}^{\top}
$$

where $\mathbf{U}$ and $\mathbf{V}$ are the matrices of left and right singular vectors, each with $r$ orthonormal columns, and $\Gamma$ is the diagonal matrix of positive singular values in decreasing order of magnitude: $\gamma_{1} \geq \cdots \geq \gamma_{r}>0$. The Eckart-Young theorem (Eckart and Young, 1936) states that if one calculates the $n \times p$ matrix $\hat{\mathbf{Z}}$ using the first $r^{*}$ singular values and corresponding singular vectors, for example for $r^{*}=2$ :

$$
\hat{\mathbf{Z}}=\left[\begin{array}{ll}
\mathbf{u}_{1} & \mathbf{u}_{2}
\end{array}\right]\left[\begin{array}{cc}
\gamma_{1} & 0 \\
0 & \gamma_{2}
\end{array}\right]\left[\begin{array}{ll}
\mathbf{v}_{1} & \mathbf{v}_{2}
\end{array}\right]^{\top}
$$

then $\hat{\mathbf{Z}}$ is the least-squares rank $r^{*}$ matrix approximation of $\mathbf{Z}$, that is $\hat{\mathbf{Z}}$ minimizes the fit criterion $\|\mathbf{Z}-\mathbf{Y}\|^{2}=\sum_{i} \sum_{j}\left(z_{i j}-y_{i j}\right)^{2}$ over all possible matrices $\mathbf{Y}$ of rank $r^{*}$, where $\|\cdots\|$ denotes the Frobenius matrix norm. It is this approximate matrix $\hat{\mathbf{Z}}$ which is biplotted in the lower $r^{*}$-dimensional space, called the reduced space. This biplot will be as accurate as is the approximation of $\hat{\mathbf{Z}}$ to $\mathbf{Z}$. The sum of squares of $\mathbf{Z}$ decomposes into two parts: $\|\mathbf{Z}\|^{2}=\|\hat{\mathbf{Z}}\|^{2}+\|\mathbf{Z}-\hat{\mathbf{Z}}\|^{2}$, where $\|\hat{\mathbf{Z}}\|^{2}=\gamma_{1}^{2}+\cdots \gamma_{r^{*}}^{2}$ and $\|\mathbf{Z}-\hat{\mathbf{Z}}\|^{2}=$ $\gamma_{r^{*}+1}^{2}+\cdots \gamma_{r}^{2}$ and goodness-of-fit is measured by the proportion of explained sum of squares $\left(\gamma_{1}^{2}+\cdots \gamma_{r^{*}}^{2}\right) /\left(\gamma_{1}^{2}+\cdots \gamma_{r}^{2}\right)$, usually expressed as a percentage.

The SVD also provides a decomposition which is a natural choice for the biplot. For example, from (3) in two dimensions, $\hat{\mathbf{Z}}=\mathbf{F G}^{\mathbf{T}}$ with

$$
\mathbf{F}=\left[\begin{array}{ll}
\gamma_{1}^{\alpha} \mathbf{u}_{1} & \gamma_{2}^{\alpha} \mathbf{u}_{2}
\end{array}\right] \quad \mathbf{G}=\left[\begin{array}{ll}
\gamma_{1}^{1-\alpha} \mathbf{v}_{1} & \gamma_{2}^{1-\alpha} \mathbf{v}_{2}
\end{array}\right]
$$

for some constant $\alpha$. The most common choices of $\alpha$ are the values 1 or 0 , when the singular values are assigned entirely either to the left singular vectors of $\mathbf{U}$ or to the right singular vectors of $\mathbf{V}$ respectively, or 0.5 when the square roots of the singular values are split equally between left and right singular vectors. Each choice, while giving exactly the same matrix approximation, will highlight a different aspect of the data matrix. The term "principal coordinates" refers to the singular vectors scaled by the singular values (for example, $\mathbf{F}$ with $\alpha=1$, or $\mathbf{G}$ with $\alpha=0$ ), while "standard coordinates" are the unscaled singular vectors (Greenacre, 1984). 
The most common biplot is of an individuals-by-variables data matrix $\mathbf{X}$ that has been transformed by centring with respect to column means $\bar{x}_{j}$ :

$$
z_{i j}=x_{i j}-\bar{x}_{j}
$$

Optionally, if normalization of the variables is required, there can be a further division of each column of the matrix by $s_{j}$, the estimated standard deviation of the $j$-th variable: $z_{i j}=\left(x_{i j}-\bar{x}_{j}\right) / s_{j}$.

After calculating the SVD of $\mathbf{Z}$, the coordinate matrices $\mathbf{F}$ and $\mathbf{G}$ are calculated as in (4) using either (i) $\alpha=1$, that is rows in principal coordinates and columns in standard coordinates, called the form biplot, which favours the display of the individuals (see below), or (ii) $\alpha=0$, that is rows in standard coordinates and columns in principal coordinates, called the covariance biplot, which favours the display of the variables (Greenacre and Underhill, 1982). In either biplot we conventionally depict the variables by rays emanating from the origin, since both their lengths and directions are important to the interpretation. Clearly, the row and column solutions in each of these biplots differ only by scale changes along the horizontal and vertical axes of the display.

\section{Insert Figure 1 about here}

The covariance biplot is characterized by the least-squares approximation of the covariance matrix $\mathbf{S}=\mathbf{Z}^{\top} \mathbf{Z} /(n-1)$ by $\mathbf{G G}^{\top} /(n-1)$, the matrix of scalar products between the row vectors of $\mathbf{G} / \sqrt{ }(n-1)$. Thus the lengths of the rays will approximate the standard deviations of the respective variables and angles between rays will have cosines which estimate the intervariable correlations. Distances between row points in the full space are measured in the Mahalanobis metric, using the inverse covariance matrix $\mathbf{S}^{-1}$. Geometrically this means that row points have been "sphered" to have the same variance in all directions.

In the form biplot, it is the form matrix $\mathbf{Z Z}^{\top}$, or matrix of scalar products between the rows of $\mathbf{Z}$, that is approximated optimally by the corresponding form matrix $\mathbf{F F}^{\top}$ of F. Thus the scalar products and squared norms (lengths) of the row vectors in the 
full space are approximated optimally in the reduced space biplot, whereas now the rays corresponding to the variables have been sphered.

Apart from these rules of interpretation summarized in Figure 1 (see, for example, Gabriel, 1971, 1981; Greenacre and Underhill, 1982; Gower and Hand, 1996), there are the lesser-known issues of calibration, approximation of differences and modelling that are particularly relevant to our study of compositional biplots.

\subsection{Calibration of biplots}

The oblique axis through a ray is called the biplot axis of the corresponding variable. Each $z_{i j}$ is approximated by the scalar product between a row point and a column point in the biplot, and this scalar product is equal to the projection of the row point onto the biplot axis, multiplied by the length of the ray. It follows that the inverse of the length of the ray gives the length of a unit along the biplot axis. For example, if the length of ray $A$ is equal to 5 , according to the scale of the display, then $1 / 5=0.2$ will be the length of one unit along this axis, so that two individuals projecting at a distance of 0.2 apart on this axis are predicted to be $0.2 \times 5=1$ unit apart on variable $A$. Knowledge of (i) this unit length, (ii) the positive direction of the scale as indicated by the ray and (iii) the fact that the mean is at the centre of the display, allows us to calibrate the biplot axis in units of the original variable. For examples of calibration, see Gabriel and Odoroff (1990), Greenacre (1993) and Gower and Hand (1996).

\subsection{Difference axes}

Any linear combination of rays in the biplot provides a vector which represents the corresponding linear combination of the variables (Gabriel, 1978). In particular, the difference between two variables can be indicated by the vector connecting the endpoints, or apexes, of the two corresponding rays (Figure 2). These difference vectors are called links. Thus, the difference between variables $A$ and $B$ is shown by the dashed link in Figure 2. Because the link points towards variable $A$, the represented difference is variable $A$ minus variable $B$. 
Insert Figure 2 about here

The row points can be similarly projected onto an axis through a link to obtain approximations of the differences for the individuals. The point of average difference on this difference axis is given by the projection of the origin onto this axis. In the covariance biplot the rays are optimal representations of the corresponding full-space columns, but there is no explicit least-squares approximation by the links to the true differences, so that error of estimation of the differences is not necessarily minimized. Differences will be accurately represented and predicted when the fit is high, of course, but when it is low differences are often represented much better with respect to radically different dimensions of the variable space. For a discussion of this topic and an explicit analysis of differences, see Greenacre (2001).

The situation is different for the relative variation biplot, however. As we shall explain in Section 3, this biplot involves a row-centred data matrix, which gives the property that links are optimal least-squares approximations of the true differences, explaining the same percentage of variance as the rays do.

\subsection{Diagnosis of simple models}

Bradu and Gabriel (1978) give guidelines for diagnosing simple models from straight-line patterns formed by subsets of row and/or column points in a two-dimensional biplot, assuming that the biplot gives an excellent fit to the data (see also Gabriel (1981)). For example, if in a biplot we observe that a subset $I$ of row points lies in an approximate straight line, and a subset $J$ of column points also lies in a straight line which is perpendicular to the line of row points, then the submatrix formed by the rows $I$ and columns $J$ can be diagnosed to follow closely the simple additive model: $z_{i j}=\mu+\alpha_{i}+\beta_{j}$. When these straight lines are not perpendicular, a slightly more general model is indicated, and slightly more general still when just one set of points, say the column points, falls on a straight line. The beauty of such diagnostics is that it is easier to notice groups of points lining up in a biplot than to undertake a study of all subsets of the points and all 
submatrices of the data.

We shall illustrate the types of model that can be diagnosed for compositional data from straight-line patterns in the display.

\section{Compositional data}

By the very nature of the initial centring transformation (5), the biplots described above apply to interval-scale variables, since the results are invariant with respect to additive changes in the variables. If the data were ratio-scale measurements, that is if multiplicative differences were important in the comparison of individuals, then the data should be logarithmically transformed before centring. We now consider compositional data and transformations which can be considered suitable to bring them onto an interval scale for biplotting.

A compositional data matrix $\mathbf{X}$ has columns corresponding to the parts, or components, of a $p$-part composition. A typical row vector of this matrix is $\left[\begin{array}{lll}x_{1} & \ldots & x_{p}\end{array}\right]$ with positive components subject to the unit-sum constraint $x_{1}+\cdots+x_{p}=1$. Although standard statistical methodology, such as the calculation of covariances and correlations, is commonly applied to compositional data, there is an extensive literature of the pitfalls of such practice (see, for example, Aitchison 1986, chap. 3). Of particular importance in the study of compositional data is the concept of a subcomposition, and the requirement that any form of analysis should possess what is called subcompositional coherence. This is best considered in terms of two scientists $\mathrm{A}$ and $\mathrm{B}$, with $\mathrm{A}$ able to record all the $p$ parts of a composition and so arrive at the full composition $\left[x_{1} \ldots x_{p}\right]$, whereas $\mathrm{B}$ is aware of, or can record, only some parts, say $1, \ldots, p^{*}$, hence arriving at the subcomposition

$$
\left[\begin{array}{lll}
s_{1} & \ldots & s_{p^{*}}
\end{array}\right]=\left[\begin{array}{lll}
x_{1} & \ldots & x_{p^{*}}
\end{array}\right] /\left(x_{1}+\cdots+x_{p^{*}}\right) .
$$

Subcompositional coherence requires that any inference which scientist A makes about the subcompositional parts $1, \ldots, p^{*}$ from knowledge of the full composition should co-

incide with the corresponding inference made about these parts by scientist $\mathrm{B}$ from the 
subcomposition. Regular product-moment correlations and principal component analysis, based on the covariances calculated on the raw compositional data, do not have subcompositional coherence (Aitchison 1986, Section 3.3).

Recognition that the study of compositions is concerned with relative and not absolute magnitudes of the components has led to considering ratios of the components. From (6) ratios are invariant under the formation of subcompositions: $s_{j} / s_{j^{\prime}}=x_{j} / x_{j^{\prime}}$. Notice that these are ratios within the compositional data vector, that is across the columns of the data matrix. When it comes to calculating scalar products and covariances for the biplot it is necessary to consider on what scale these ratios themselves are, when compared across individuals. Here we maintain that the ratios themselves are on a ratio scale. Hence it is appropriate to take logarithms of the ratios and to consider differences between these logratios from individual to individual. Several different justifications for the logratio transformation may be found in Aitchison (1986, 2001). At first, this might seem unduly complicated but differences in logratios are already commonplace in the calculation of the log-odds in the loglinear model of categorical data and in logistic regression.

Aitchison (1986) shows that there are three equivalent ways of considering ratios within a compositional vector: $(1)$ the $\frac{1}{2} p(p-1)$ ratios $x_{j} / x_{j^{\prime}}$ between pairs of components (we assume $j<j^{\prime}$ when selecting the pair), (2) the $p-1$ ratios $x_{j} / x_{p}$ between the first $p-1$ components and the last one, and (3) the $p$ ratios $x_{j} / g(\mathbf{x})$ between the components and their geometric average $g(\mathbf{x})=\left(x_{1} x_{2} \cdots x_{p}\right)^{1 / p}$. On the logarithmic scale these are the differences $\log \left(x_{j}\right)-\log \left(x_{j^{\prime}}\right), \log \left(x_{j}\right)-\log \left(x_{p}\right)$ and the deviations from the mean $\log \left(x_{j}\right)-(1 / p) \sum_{j} \log \left(x_{j}\right)$ respectively. The second option is the least interesting in the present context, because it is not symmetric with respect to all the components, and we do not discuss it further. We shall be primarily interested in the study of pairwise $\log$ ratios $\log \left(x_{j} / x_{j^{\prime}}\right)=\log \left(x_{j}\right)-\log \left(x_{j^{\prime}}\right)$, but will need to refer to the centred $\operatorname{logratios}$ $\log \left[\left(x_{j}\right) / g(\mathbf{x})\right]$ as well.

Suppose that we denote the $\log a r i t h m s ~ \log \left(x_{i j}\right)$ of the compositional data matrix by $\ell_{i j}$ and collect them in a matrix $\mathbf{L}(n \times p)$. Suppose that the dot subscripts in $\ell_{i}, \ell_{\cdot j}$ and $\ell$.. denote the averages over the corresponding indices. Also define $\tau_{i, j j^{\prime}}=\ell_{i, j}-\ell_{i j^{\prime}}$ as 
the general element of the $n \times \frac{1}{2} p(p-1)$ matrix $\mathbf{T}$ of all pairwise logratios, where $j<j^{\prime}$. Although our interest is chiefly in the matrix $\mathbf{T}$ of logratios, we shall now show that it is possible to obtain all the results about $\mathbf{T}$ using a smaller matrix with only $p$ columns based on the centred logratios.

If we were to biplot the larger matrix $\mathbf{T}$, we would centre $\mathbf{T}$ with respect to column means $\tau_{\cdot j j^{\prime}}=\ell_{\cdot j}-\ell_{\cdot j^{\prime}}$, as in (5), to obtain a matrix $\mathbf{Y}: y_{i, j j^{\prime}}=\tau_{i, j j^{\prime}}-\tau_{\cdot j j^{\prime}}$. Suppose that $\mathbf{Y}$ has SVD $\mathbf{Y}=\mathbf{A} \Psi \mathbf{B}^{\mathbf{\top}}$, where $\mathbf{B}$ has $\frac{1}{2} p(p-1)$ rows representing each logratio $\left(j j^{\prime}\right)$ as a ray emanating from the origin. Notice that the corresponding "inverse" logratio $\left(j^{\prime} j\right)$ would be the ray of the same length emanating from the origin and pointing in the opposite direction. $\mathbf{T}$ has $\frac{1}{2} p(p-1)$ columns, but its rank can be shown to be equal to $p-1$, hence it has $\frac{1}{2}(p-1)(p-2)$ columns that are effectively redundant.

Considering now the analysis of the centred logratios, let $\mathbf{Z}$ be the $n \times p$ matrix of centred $\operatorname{logratios} \ell_{i j}-\ell_{i}$. centred with respect to column means $z_{\cdot j}=\ell_{\cdot j}-\ell \ldots$ That is, $\mathbf{Z}$ is the matrix of elements of $\mathbf{L}$ double-centred: $z_{i j}=\ell_{i j}-\ell_{i}-\ell_{. j}+\ell \ldots$ Let $\mathbf{Z}$ have SVD $\mathbf{Z}=\mathbf{U} \Gamma \mathbf{V}^{\top}$. Since $\mathbf{Z}$ is double-centred, its singular vectors in $\mathbf{U}$ and $\mathbf{V}$ are all centred, and the rank of $\mathbf{Z}$ is equal to $p-1$.

The SVDs of $\mathbf{Y}$ and of $\mathbf{Z}$ are directly related in the following way (see Appendix 1 for a proof of these results):

1. The singular values of the two SVDs are related by a constant scaling factor: $\Psi=$ $\sqrt{ } p \Gamma$.

2. The left singular vectors are identical: $\mathbf{A}=\mathbf{U}$.

3. The right singular vectors $\mathbf{B}$ of $\mathbf{Y}$ are proportional to the corresponding differences in the row vectors of $\mathbf{V}$, specifically $b_{j j^{\prime}, k}=\left(v_{j k}-v_{j^{\prime} k}\right) / \sqrt{ } p$.

This result means that we need only perform the analysis of the smaller matrix $\mathbf{Z}$, from which all the results for the larger matrix $\mathbf{Y}$ may be obtained. We call the biplot of $\mathbf{Z}$ the "relative variation biplot" because it represents variation in all the component ratios. Important geometric consequences come from the equivalence of the SVDs of $\mathbf{Y}$ and $\mathbf{Z}$. 
First, in the relative variation biplot we obtain rays representing the centred logratios. The links between the apexes of the rays represent the pairwise logratios and can effectively be transferred to the origin to obtain the solution which would have been obtained from Y. This means that looking for straight-line patterns in the biplot can be widened to include links which are parallel. Two parallel links of the same length, thus forming a parallelogram, will be shown to have a very special property in the relative variation biplot. Second, we can be assured that in the relative variation biplot, the pairwise logratios are optimally displayed and with the same percentage explained variance as the display of the centred logratios. This result is due to the row-centring of the log-compositional matrix $\mathbf{L}$, which assures that column links are optimal representations, just as the column-centring of a regular individuals-by-variables matrix assures that the distances between individuals are also optimally displayed.

We shall illustrate this novel result along with other features of the relative variation biplot.

\section{Results}

Figures 3 and 4 show the relative variation biplots of the data in Table 1, first the form biplot version and second the covariance biplot version. In the logratio covariance biplot

of Figure 4 the column points have been rescaled by the constant $1 / \sqrt{ }(n-1)=1 / \sqrt{ } 21$ in order to bring the column solution onto the scale of logratio variance and covariance.

\section{Insert Figures 3 and 4 about here}

We collect below the properties of the relative variation biplots. The following notation will be used when referring to features of the biplots: $O$ refers to the origin of the displays; $i$ denotes the $i$-th row point in standard coordinates, $I$ the $i$-th row point in principal coordinates; similarly, $j$ and $J$ denote the $j$-th column point in standard and principal coordinates, so that the form biplot displays the I's and $j$ 's while the covariance biplot displays the $i$ 's and $J$ 's; vectors such as rays and links are indicated by the endpoints, for 
example in the covariance biplot $O J$ is the ray to the $j$-th component apex and $J J^{\prime}$ is the link from the $j$-th to the $j^{\prime}$-th apexes, representing the difference $J^{\prime}$ minus $J$; distances between points are denoted by, for example, $|O I|,\left|J J^{\prime}\right|$ and $\left|i i^{\prime}\right|$.

Property 1. The row points and column points are both centred at the origin $O$. This is a direct consequence of the double-centring transformation of the matrix. Thus the average row point in the display is at the origin and the average column point as well.

Property 2. Distances $\left|I I^{\prime}\right|$ between row points in the form biplot are approximations of the distances between the individuals, calculated either from the matrix of centred logratios, or equivalently from the matrix of pairwise logratios. The dispersion along the horizontal and vertical principal axes is quantified by the corresponding eigenvalues and percentages of sum of squares explained: $90.0 \%$ and $8.2 \%$ respectively in this application, giving an excellent overall fit of $98.2 \%$.

Property 3. Distances $\left|J J^{\prime}\right|$ between column points in the covariance biplot are approximations of the standard deviation of the corresponding logratio. Thus the short link between "black" and "other" indicates that the component ratio black/other is relatively constant in the data, whereas the largest link, between "red" and "blue", indicates that there is the most relative variation in these two colours across the paintings. The exact standard deviations of all logratios are given in the upper triangle of Table 2 and those estimated from the link lengths in the biplot in the lower triangle. The agreement is very good because the biplot fits the data so well. Notice that the estimated values are always less than the exact values, since the approximation is "from below". The link lengths in the full five-dimensional space are exactly the standard deviations, but are shorter when projected onto the reduced space of the biplot.

\section{Insert Table 2 about here}

Property 4. Angle cosines between links in the covariance biplot estimate correlations between logratios. Thus the fact that the links among blue, yellow and red lie perpendicular to the links between white, other and black, indicates that logratios amongst the 
first set have near zero correlations with those amongst the second set. To support this claim, we show in Table 3 the relevant subset of the correlation matrix between logratios, showing that the two sets can be considered independent of each other.

Amongst the colours blue, yellow and red of the first set the correlations between logratios are seen to be high, as expected. Amongst those of the second set, black, white and other, however, there are lower correlations, especially between $\log$ (white/other) and $\log$ (other/black). This is due to the fact that "other" has a relatively large coordinate on the third dimension which is not seen in the two-dimensional biplot. The black-other link, although short and thus relatively unimportant to the interpretation, is not well represented in the biplot. This fact can also be picked up on closer inspection of Table 2, where the standard deviation of the black-other logratio is seen to be underestimated in the biplot.

\section{Insert Table 3 about here}

Property 5. In either biplot column points lying in a straight line reveal logratios of high correlation, and a model summarizing this interdependency can be deduced from the relative lengths of their links. By inspection, the distance from red to yellow is roughly 2.5 times the distance from yellow to blue. Since all links can be transferred to the origin, it follows that

$$
\log (\text { red } / \text { yellow })-\operatorname{ave}\{\log (\text { red } / \text { yellow })\}=2.5[\log (\text { yellow/blue })-\operatorname{ave}\{\log (\text { yellow/blue })\}]
$$

where ave $(\cdots)$ indicates the mean of the corresponding logratio across individuals. This reduces to the constant logcontrast

$$
2.5 \log (\text { blue })+\log (\text { red })-3.5 \log (\text { yellow })=\text { constant }
$$

where the constant is estimated by averaging the logcontrast over individuals. This diagnoses a proportionality relationship between the colours as

$$
\frac{\text { red }}{\text { yellow }} \propto\left(\frac{\text { yellow }}{\text { blue }}\right)^{2.5} \text {. }
$$


Figure 5 demonstrates this proportionality relationship while Figure 6 shows the relationship in triangular coordinates between the three primary colours for the 3-part subcomposition, showing an excellent fit to the data. Interestingly, this representation of primary colours as vertices of a triangle is due to Goethe (1810), and is the earliest reference, to our knowledge, to the triangular coordinate system. The same system was used independently 50 years later by Maxwell (1860) to explain his theory of colours in terms of red, green and blue.

\section{Insert Figures 5 and 6 about here}

In general, if three components $\mathrm{A}, \mathrm{B}$ and $\mathrm{C}$ lie in an approximate straight line with distances $\mathrm{AB}$ and $\mathrm{BC}$ equal to $\lambda$ and $\mu$ respectively, then the constant logcontrast is of the form $\mu \log (\mathrm{A})+\lambda \log (\mathrm{C})-(\lambda+\mu) \log (\mathrm{B})=$ constant, that is $(\mathrm{A} / \mathrm{B})^{\mu} \propto(\mathrm{B} / \mathrm{C})^{\lambda}$.

Property 6. In either biplot four column points A, B, C and D forming a parallelogram reveal a simple constant logcontrast of the form

$$
\log (\mathrm{A})-\log (\mathrm{B})+\log (\mathrm{C})-\log (\mathrm{D})=\text { constant. }
$$

In Figures 3 and 4 the colours black, red, white and blue lie approximately on a parallelogram. We can transfer the links black-red and blue-white to the origin and thus obtain the relationship

$$
\log (\text { black} / \text { red })-\operatorname{ave}\{\log (\text { black } / \text { red })\}=\log (\text { blue/white })-\text { ave }\{\log (\text { blue/white })\}
$$

leading to the constant logcontrast

$$
\log (\text { black })-\log (\text { red })+\log (\text { white })-\log (\text { blue })=\text { constant }
$$

and thus the proportionality relationship (black/red) $\propto$ (blue/white) or equivalently (black/blue) $\propto($ red/white). This relationship can be demonstrated by plotting the ratio of any two adjacent colours in the parallelogram against the ratio of the other two. Figure 7 shows black/red against blue/white and the relationship is strongly linear through the origin, as diagnosed successfully by the parallelogram in the biplot. 
Insert Figure 7 about here

Property \%. If a subset $I$ of the individuals (rows) and a subset $J$ of the components columns lie approximately on respective straight lines that are orthogonal, then the compositional submatrix formed by the rows $I$ and columns $J$ has approximately constant logratios amongst the components, that is the double-centred submatrix of $\log$ (compositions) has near-zero entries. For example in both biplots, it is possible to see a group of three row points in the lower left quadrant (rows 9, 21 and 15 respectively) which are in a straight line orthogonal to the line defined by the three column points white-other-black. Table 4 shows the relevant submatrix of Table 1 and the three logratios, which are confirmed to be fairly constant over the rows, with slightly more variability in ratios involving "other" which has already been seen to be not poorly represented in the two-dimensional biplot.

\section{Insert Table 4 about here}

This property of logratio constancy in submatrices of the data can be deduced directly from the additive model mentioned in Section 2.3 or from the concept of biplot calibration, illustrated by the next property.

Property 8. Either biplot can be calibrated in logratio units and thus in ratio units. We are mostly interested in the links, so let us take the link from red to yellow as an example and calibrate the biplot axis through this link in the covariance biplot. It will be easier to illustrate the calibration in logratio units first, since this is linear on the biplot axis.

From Table 2 the length of the yellow-red link will have length equal to 0.767. Thus one unit on its logratio scale will have length equal to $1 / 0.767=1.304$ and a 0.1 unit will have length 0.1304 . The mean value of $\log ($ yellow/red) is calculated from the data to be 1.073 , which is the value corresponding to the origin of the display projected onto this link. So in order to calibrate this axis on a scale of tenths $(0.1)$ of a unit, we have to put tic marks on the axis connecting red to yellow, at a distance 0.1304 apart, so that the 
scale increases towards the right and has the value 1.073 at the point where the origin projects onto the biplot axis. Equivalently, we can transfer the link to the origin in which case the origin will correspond to the average logratio.

The trigonometry needed to calculate the tic marks for a biplot axis through a link or a ray is given in Appendix 2, and the result is illustrated in Figure 8, for logratios $\log$ (yellow/red) and $\log$ (white/black). Because the white/black link is shorter than the yellow/red one, a $\log$ (white/black) unit difference is longer and so the tic marks are more spread out, which is another way of saying that the dispersion is less. Any row point can now be projected onto these biplot axes and the corresponding value of the logratio can be estimated. The estimates will be generally very good, because the quality of the display is high (98.2\%). A ray can be calibrated in exactly the same way, although the interpretation of centred logratios is more complex than pairwise logratios.

\section{Insert Figure 8 about here}

Calibration gives the biplot a concrete interpretation in terms of the original data and provides a new meaning to some of the properties already stated. For example, property 7 is obvious now since any points lying on a line perpendicular to a link project onto the same value on its biplot axis and thus have constant estimated values of the corresponding logratios.

We could similarly calibrate the form biplot, in fact this is the biplot of choice for calibration. Since the column points are sphered, it is now the shape of the row points which indicates the spread in a more visually obvious way, hence the term "form" biplot.

It is also possible to calibrate either biplot in units of ratios, not logratios. These tic marks are now not equidistant on the biplot axis, but the scale is now closer to the original data. Figure 9 shows the form biplot calibrated in ratio units, for the same colour ratios as before.

\section{Insert Figure 9 about here}

Property 9. The whole compositional data matrix can be reconstructed approximately 
from either biplot, but we need to know the means of the centred logratios as well as the geometric means of the rows to be able to "uncentre" the estimates obtained from the biplot. As before we calibrate each ray representing the centred logratio of a colour, for which we need to know the average centred logratio to be able to anchor the scale at the origin. Then projecting each painting $i$ onto each colour axis $j$ we obtain the estimate of the centred logratio $\log \left[x_{i j} / g\left(\mathbf{x}_{i}\right)\right]$, and with knowledge of the geometric mean $g\left(\mathbf{x}_{i}\right)$ of the row we can eventually arrive at an estimate of $x_{i j}$ itself. The reconstructed data from the two-dimensional biplot are given in Table 5, and are seen to be very close to the original data, to be expected from the $98.2 \%$ explained variance in the biplot.

\section{Discussion}

The present approach is based on a certain choice of prerequisites which a method of compositional data analysis should reasonably be expected to satisfy. Most importantly, the unit-sum constraint - or equivalently the fact that all compositional data vectors occupy a simplex space - should be respected throughout the analysis, and all results should have subcompositional coherence. It is clear from the above aspects of interpretation that the fundamental elements of a relative variation biplot are the links, rather than the rays as in the usual case of biplots. The complete set of links, specifying the relative variances, determines the compositional covariance structure and provides direct information about subcompositional variability and independence.

The relative variation biplot implies a certain metric, or distance function, between sample points $i$ and $i^{\prime}$. As we have seen in Section 3, the squared distance can be defined either in terms of all $\frac{1}{2} p(p-1)$ logratios, or - more parsimoniously - in terms of the $p$ centred logratios:

$$
\begin{aligned}
d_{i i^{\prime}}^{2} & =\frac{1}{p} \sum \sum_{j<j^{\prime}}\left(\log \frac{x_{i j}}{x_{i j^{\prime}}}-\log \frac{x_{i^{\prime} j}}{x_{i^{\prime} j^{\prime}}}\right)^{2} \\
& =\sum_{j}\left(\log \frac{x_{i j}}{g\left(\mathbf{x}_{i}\right)}-\log \frac{x_{i^{\prime} j}}{g\left(\mathbf{x}_{i^{\prime}}\right)}\right)^{2}
\end{aligned}
$$

This metric satisfies the property that the distance between any two compositions must 
be at least as great as the distance between any corresponding subcompositions of the compositions. For an account of how to determine an appropriate metric for compositional vectors, see Aitchison (1992). A study of the drawbacks of other metrics in the simplex space is reported by Martín-Fernández et al (1998).

Attempts have been made, for example by Miesch et al (1966), David et al (1974), Teil and Cheminée (1975), to explore compositional variability through the use of singular value decompositions based on the raw or standardized compositional data. These approaches do not recognize specifically the compositional nature of the data and do not have the property of subcompositional coherence. Reconstruction of compositional vectors using biplots based on correspondence analysis (Benzécri, 1973), for example, can sometimes lead to estimated components that are negative, hence outside the simplex.

As far as identifying relationships between the components $x_{j}$ of a composition is concerned, straight or parallel line patterns in the relative variation biplot indicate a particular class of models that can be written as a constant logcontrast: $\sum_{j} a_{j} \log \left(x_{j}\right)=$ constant, where $\sum_{j} a_{j}=0$. Constant logcontrast relationships are important in many disciplines, for example the Hardy-Weinberg equilibrium in population genetics (Hardy, 1908) is a constant logcontrast in the gene frequencies, and various equilibrium equations in geochemistry also reduce to constant logcontrasts (Krauskopf, 1979); see also Aitchison (1999) for further discussion of logcontrast laws. It can be argued that constant logcontrasts do not cover all compositional relationships of possible interest, but this is no different from the situation with the regular biplot in which only a certain class of models can be diagnosed from straight-line patterns in the display.

The biplot is a natural consequence of the singular value decomposition of a matrix. To use standard singular value decomposition technology, defined on conventional multidimensional vector spaces, the compositional data are log-transformed and then doublecentred to ensure that component ratios are analyzed on a ratio scale. Even though the initial log-transformation takes the data out of the simplex into unconstrained real vector space, the compositional nature of the data vectors is respected throughout the analysis. Aitchison et al. (2001) shows that exactly the same methodology can be described 
equivalently by a singular value decomposition which is defined directly in terms of compositions in the constrained simplex space. The simplex is established as a vector space in its own right using compositional group operators of addition and scalar multiplication. The addition operation in this "stay-in-the-simplex", or simplicial, approach is called perturbation, denoted by $\oplus$, and scalar multiplication is called powering, denoted by $\otimes$. Without going into details about these operations, we can use them to reconstruct the $i$-th row $\mathbf{x}_{i}$ of the compositional data matrix in the following way, analogous to principal component analysis:

$$
\mathbf{x}_{i}=\boldsymbol{\xi} \oplus\left(s_{1} u_{i 1} \otimes \boldsymbol{\beta}_{\mathbf{1}}\right) \oplus \cdots\left(s_{r} u_{i r} \otimes \boldsymbol{\beta}_{\boldsymbol{r}}\right)
$$

where $\boldsymbol{\xi}$ is the compositional centre of the data set, the $s_{k}$ 's are positive "singular values", the $\boldsymbol{\beta}_{\boldsymbol{k}}$ 's the "right singular vectors" which form a compositional basis in the simplex, thus providing the "principal axes" of the data compositions, and $s_{k} u_{i k}$ the "principal coordinates" with respect to the simplicial basis. For our colour data, the first two simplicial basis vectors turn out to be:

$\boldsymbol{\beta}_{\mathbf{1}}=\left[\begin{array}{lll}0.1560 .1490 .0850 .3330 .1250 .153\end{array}\right]^{\top} \quad \boldsymbol{\beta}_{\mathbf{2}}=\left[\begin{array}{lll}0.0880 .3120 .1700 .1730 .1550 .103\end{array}\right]^{\top}$.

The way to interpret these compositional basis vectors is - as before - to look at ratios between their components. Thus the constancy of the black, white and other values (first, second and sixth) in $\boldsymbol{\beta}_{\mathbf{1}}$ shows that this subcomposition is stable in the first simplicial "dimension", while the constancy of blue, red and yellow (third, fourth and fifth values) in $\boldsymbol{\beta}_{\mathbf{2}}$ shows a similar stability of this subcomposition in the second dimension.

Finally, we have been using the classical form of the biplot, now often referred to as the linear biplot since the definition of nonlinear biplots by Gower and Harding (1988). In nonlinear biplots the biplot axes are replaced by curved trajectories and can also be calibrated. This richer but more complex biplot can possibly identify a wider class of relationships, but its potential still needs to be fully explored. 


\section{Acknowledgments}

We would like to express thanks to John Birks and Richard Reyment for valuable discussion in the earlier stages of this work. Cajo ter Braak and John Gower gave useful comments on an earlier version of this paper submitted for publication. Rosemarie Nagel and Kic Udina made valuable comments and pointed out the interesting references to Johann Wolfgang von Goethe and James Clerck Maxwell respectively, whose colour theories were both based on triangular coordinates. Michael Greenacre's research is supported by Spanish Ministry of Science and Technology grant number BFM2000-1064.

\section{References}

Aitchison, J. (1986) The Statistical Analysis of Compositional Data. London: Chapman and Hall.

Aitchison, J. (1992) On criteria for measures of compositional difference. Math. Geol., 22, 223-226.

Aitchison, J. (1999) Logratios and natural laws in compositional data analysis. Math. Geol., 31, 563-589.

Aitchison, J. (2001) Simplicial inference. In Algebraic Structures in Statistics, Contemporary Mathematics Series of the American Mathematical Society (ed. M. Viana and D. Richards), to appear.

Aitchison, J., Barceló-Vidal, C., Martín-Fernández, J. A. and Pawlowsky-Glahn, V. (2001) Reply to Letter to the Editor by S. Rehder and U. Zier on "Logratio analysis and compositional distance" by J. Aitchison, C. Barceló-Vidal, J. A. Martín-Fernández and V. Pawlowsky-Glahn. Math. Geol., 33, to appear.

Benzécri, J.-P. (1973), L'Analyse des Données. Tome I: La Classification. Tome II: l'Analyse des Correspondances, Paris: Dunod.

Bradu, D. and Gabriel, K. R. (1978) The biplot as a diagnostic tool for models of two-way tables. Technometrics, 20, 47-68.

David, M., Campiglio, C. and Darling, R. (1974) Progresses in R- and Q-mode analy- 
sis: correspondence analysis and its application to the study of geological processes. Can. J. Earth Sci., 11, 131-146.

Eckart, C. and Young, G. (1936) The approximation of one matrix by another of lower rank. Psychometrika, 1, 211-218.

Gabriel, K. R. (1971) The biplot-graphic display of matrices with application to principal component analysis. Biometrika, 58, 453-467.

Gabriel, K. R. (1978) Analysis of meteorological data by means of canonical decomposition and biplots. J. Appl. Meteorology, 11, 1072-1077.

Gabriel, K. R. (1981) Biplot display of multivariate matrices for inspection of data and diagnosis. In Interpreting Multivariate Data (ed. V. Barnett), pp. 147-173. New York: Wiley.

Gabriel, K. R. and Odoroff, C. L. (1990) Biplots in biomedical research, Statistics in Medicine, 9, 469-485.

Goethe, J. W. (1810) Zur Farbenlehre. Tübingen. (http://www.colorsystem.com/projekte/engl/14goee.htm)

Gower, J. C. and Harding, S. (1988) Non-linear biplots. Biometrika, 73, 445-455.

Gower, J.C. and Hand, D. (1996) Biplots. London: Chapman and Hall.

Greenacre, M. J. (1984) Theory and Applications of Correspondence Analysis. London: Academic Press.

Greenacre, M.J. (1993) Biplots in correspondence analysis. J. Appl. Stat., 20, 251-269.

Greenacre, M.J. (2001) Analysis of matched matrices. Working report number 539, Department of Economics and Business, Universitat Pompeu Fabra, submitted for publication (http://www.econ.upf.es/cgi-bin/onepaper?539).

Greenacre, M. J. and Underhill, L. G. (1982) Scaling a data matrix in low-dimensional Euclidean space. In Topics in Applied Multivariate Analysis (ed. D. M. Hawkins), pp. 183-268. Cambridge, UK: Cambridge University Press.

Hardy, G. H. (1908) Mendelian proportions in a mixed population. Science, 28, 49-50. 
Krauskopf, K. B. (1979) Introduction to Geochemistry. New York: McGraw Hill.

Martín-Fernández, J. A., Barceló-Vidal, C. and Pawlowsky-Glahn, V. (1998) Measures of difference for compositional data and hierarchical clustering. In Proceedings of IAMG'98, The Fourth Annual Conference of the International Association for Mathematical Geology (eds. A. Baccianti, G. Nardi and R. Potenza), pp. 526-531. Naples: De Frede Editore.

Maxwell, J. C. (1860) On the theory of compound colours. Philosophical Transactions, 150, 57-84 (http://www.colorsystem. com/projekte/engl/19maxe.htm).

Miesch, A. T., Chao, E. C. T. and Cuttitta, F. (1966) Multivariate analysis of geochemical data on tektites. J. Geol., 74, 673-691.

Teil, H. and Cheminée, J.L. (1975) Application of correspondence factor analysis to the study of major and trace elements in the Erta ale Chain (Afar, Ethiopia). Math. Geol., 7, 13-30. 


\section{Appendix 1}

\section{Equivalence of logratio and centred logratio biplots}

Here we prove the result stated in Section 3. Suppose that we collect the logarithms $\ell_{i j} \equiv \log \left(x_{i j}\right)$ of the compositional data in the matrix $\mathbf{L}(n \times p)$. Then the matrix of all $\operatorname{logratios} \log \left(x_{i j} / x_{i j^{\prime}}\right)=\ell_{i j}-\ell_{i j^{\prime}}\left(\right.$ for $\left.j<j^{\prime}\right)$ is equal to $\mathbf{L E}_{p}$, where $\mathbf{E}_{p}$ is the $p \times \frac{1}{2} p(p-1)$ differencing matrix with zeros in each column except for a 1 and -1 in two rows. The matrix of centred $\log$ ratios $\log \left[x_{i j} /\left(x_{i 1} \cdots x_{i p}\right)^{1 / p}\right]$ is equal to $\mathbf{L C}_{p}$, where $\mathbf{C}_{p}$ is the $p \times p$ idempotent centring matrix $\mathbf{I}-(1 / p) \mathbf{1 1}{ }^{\top}$. Examples of the differencing and centring matrices are, for $p=4$ :

$$
\mathbf{E}_{4}=\left[\begin{array}{rrrrrr}
1 & 1 & 1 & 0 & 0 & 0 \\
-1 & 0 & 0 & 1 & 1 & 0 \\
0 & -1 & 0 & -1 & 0 & 1 \\
0 & 0 & -1 & 0 & -1 & -1
\end{array}\right] \quad \mathbf{C}_{4}=\left[\begin{array}{rrrr}
3 / 4 & -1 / 4 & -1 / 4 & -1 / 4 \\
-1 / 4 & 3 / 4 & -1 / 4 & -1 / 4 \\
-1 / 4 & -1 / 4 & 3 / 4 & -1 / 4 \\
-1 / 4 & -1 / 4 & -1 / 4 & 3 / 4
\end{array}\right]
$$

Consider the matrix $\mathbf{L} \mathbf{C}_{p}$ of centred logratios first. A biplot of this matrix as described in Section 2 would centre with respect to column means as in (5), that is premultiply by $\mathbf{C}_{n}: \mathbf{Z}=\mathbf{C}_{n} \mathbf{L} \mathbf{C}_{p}$, and then proceed as before with the SVD as in (2). The matrix $\mathbf{Z}$ is thus the double-centred matrix of $\log \left(\right.$ compositions), with elements $z_{i j}=\ell_{i j}-\ell_{i}-\ell_{\cdot j}+\ell$.. where the dot subscript indicates averaging over the corresponding index. Suppose that $\mathbf{Z}$ has SVD $\mathbf{Z}=\mathbf{U} \Gamma \mathbf{V}^{\top}$. The fact that $\mathbf{Z}$ is double-centred implies that the elements of each singular vector in $\mathbf{U}$ and $\mathbf{V}$ are centred: $\mathbf{C}_{n} \mathbf{U}=\mathbf{U}$ and $\mathbf{C}_{p} \mathbf{V}=\mathbf{V}$.

Consider now the matrix $\mathbf{L E}_{p}$ of pairwise logratios. This matrix, again centred with respect to column means, gives $\mathbf{Y}=\mathbf{C}_{n} \mathbf{L} \mathbf{E}_{p}$ and leads to a biplot which depicts the individuals and each $\left(j, j^{\prime}\right)$ ratio pair $\left(j<j^{\prime}\right)$. Suppose $\mathbf{Y}$ has $\operatorname{SVD} \mathbf{Y}=\mathbf{A} \Psi \mathbf{B}^{\top}$, where B has $\frac{1}{2} p(p-1)$ rows.

These two biplots are directly related through the SVDs as follows. Firstly, the form matrices of $\mathbf{Z}$ and $\mathbf{Y}$ are identical, apart from an overall scale factor

$$
\begin{gathered}
\mathbf{Z Z}^{\top}=\mathbf{C}_{n} \mathbf{L} \mathbf{C}_{p} \mathbf{C}_{p} \mathbf{L}^{\top} \mathbf{C}_{n}=\mathbf{C}_{n} \mathbf{L} \mathbf{C}_{p} \mathbf{L}^{\top} \mathbf{C}_{n}=\mathbf{U} \Gamma^{2} \mathbf{U}^{\top} \\
\mathbf{Y} \mathbf{Y}^{\top}=\mathbf{C}_{n} \mathbf{L} \mathbf{E}_{p} \mathbf{E}_{p}^{\top} \mathbf{L}^{\top} \mathbf{C}_{n}=p \mathbf{C}_{n} \mathbf{L} \mathbf{C}_{p} \mathbf{L}^{\top} \mathbf{C}_{n}=\mathbf{U}\left(p \Gamma^{2}\right) \mathbf{U}^{\top}
\end{gathered}
$$


since $\mathbf{E}_{p} \mathbf{E}_{p}^{\top}=p \mathbf{C}_{p}$. Thus the singular values differ by a constant scale factor of $\sqrt{ } p$ : $\Psi=\sqrt{ } p \Gamma$ and the left singular vectors are identical in the two SVDs: $\mathbf{A}=\mathbf{U}$. On the other hand, the scalar products of the columns, which provide the covariances in the two biplots, have the following connection.

$$
\mathbf{Z}^{\top} \mathbf{Z}=\mathbf{C}_{p} \mathbf{L}^{\top} \mathbf{C}_{n} \mathbf{L} \mathbf{C}_{p}=\mathbf{V} \Gamma^{2} \mathbf{V}^{\top}
$$

Pre- and postmultiplying by $\mathbf{E}_{p}^{\top}$ and $\mathbf{E}_{p}$ respectively and using the fact that the columns of $\mathbf{E}_{p}$ are centred: $\mathbf{C}_{p} \mathbf{E}_{p}=\mathbf{E}_{p}$, we obtain

$$
\mathbf{Y}^{\top} \mathbf{Y}=\mathbf{E}_{p}^{\top} \mathbf{L}^{\top} \mathbf{C}_{n} \mathbf{C}_{n} \mathbf{L} \mathbf{E}_{p}=\left(\mathbf{E}_{p}^{\top} \mathbf{V}\right) \Gamma^{2}\left(\mathbf{E}_{p}^{\top} \mathbf{V}\right)^{\top}
$$

that is, the right singular vectors of $\mathbf{B}$ are proportional to the corresponding differences between rows of $\mathbf{V}$. Since $\left(\mathbf{E}_{p}^{\top} \mathbf{V}\right)^{\top}\left(\mathbf{E}_{p}^{\top} \mathbf{V}\right)=\mathbf{V}^{\top} \mathbf{E}_{p} \mathbf{E}_{p}^{\top} \mathbf{V}=\mathbf{V}^{\top}\left(p \mathbf{C}_{p}\right) \mathbf{V}=p \mathbf{V}^{\top} \mathbf{V}=p \mathbf{I}$ it follows that $\mathbf{B}=\mathbf{E}_{p}^{\top} \mathbf{V} / \sqrt{ } p$ and we verify again that $\Psi=\sqrt{ } p \Gamma$.

With the above notation it is easy to show that, in general, a matrix $\mathbf{Y}$ (columncentred or not) has form matrix $\mathbf{Y} \mathbf{Y}^{\top}$, whereas the form matrix of its column differences $\mathbf{Y} \mathbf{E}_{p}$ is $p \mathbf{Y C}_{p} \mathbf{Y}^{\top}$. Thus the form matrices agree (up to the scale value $p$ ) if $\mathbf{Y}$ is rowcentred, but also if $\mathbf{Y}$ has constant row sums since row-centring would then just involve subtracting a constant from every matrix element. Thus a regular principal component analysis of a matrix of compositional data will also have the property that links are optimal representations of the column differences. 


\section{Appendix 2}

\section{Linear biplot calibration}

Suppose that we want to calibrate the biplot axis which passes through two column points $A$ and $B$, with given coordinates $\left(a_{1}, a_{2}\right)$ and $\left(b_{1}, b_{2}\right)$ on the first two dimensions of the biplot. Denote the projection of the origin of the biplot onto the biplot axis by the point $\left(o_{1}, o_{2}\right)$. Suppose that the mean difference in the values of $B-A$ (calculated from the data) is equal to $m$.

Now the squared distance $|A B|^{2}$ is equal to $d^{2}=\left(b_{1}-a_{1}\right)^{2}+\left(b_{2}-a_{2}\right)^{2}$ and the length of 1 unit on the biplot axis is thus $s=1 / d$. By simple trigonometry, the coordinates $\left(o_{1}, o_{2}\right)$ are equal to

$$
\begin{aligned}
& o_{1}=\left[a_{1}\left(b_{2}-a_{2}\right)^{2}-a_{2}\left(b_{1}-a_{1}\right)\left(b_{2}-a_{2}\right)\right] / d^{2} \\
& o_{2}=\left[a_{2}\left(b_{1}-a_{1}\right)^{2}-a_{1}\left(b_{1}-a_{1}\right)\left(b_{2}-a_{2}\right)\right] / d^{2}
\end{aligned}
$$

and the tic mark for value $t$ has coordinates $\left(t_{1}, t_{2}\right)$ :

$$
\begin{aligned}
& t_{1}=o_{1}+s(t-m)\left(b_{1}-a_{1}\right) / d=o_{1}+(t-m)\left(b_{1}-a_{1}\right) / d^{2} \\
& t_{2}=o_{2}+s(t-m)\left(b_{2}-a_{2}\right) / d=o_{2}+(t-m)\left(b_{2}-a_{2}\right) / d^{2}
\end{aligned}
$$

As an example, for the red-yellow link in the covariance biplot of Figure 7, the given values are the coordinates of the apexes, $\left(a_{1}, a_{2}\right)=(-0.612,0.0284)$ and $\left(b_{1}, b_{2}\right)=$ $(0.154,0.0036)$, and the mean value of $\log (\mathrm{red} /$ yellow $), m=1.073$.

The link distance is equal to 0.767 and the unit distance on the biplot axis will thus be $1 / 0.767=1.304$ The origin projected onto the biplot axis, corresponding to the mean value, has coordinates $\left(o_{1}, o_{2}\right)=(0.0003,0.0096)$ and the tic mark for the value 0.7 , for example, has coordinates:

$$
\begin{gathered}
t_{1}=0.0003+(0.7-1.073) \times(0.154+0.612) / 0.767^{2}=-0.486 \\
t_{2}=0.0096+(0.7-1.073) \times(0.0036-0.0284) / 0.767^{2}=0.0243
\end{gathered}
$$

The above formulae can be used to calibrate a ray as well by setting $\left(a_{1}, a_{2}\right)=0$. 
Figure 1

Summary of interpretation of (a) covariance biplot, (b) form biplot.

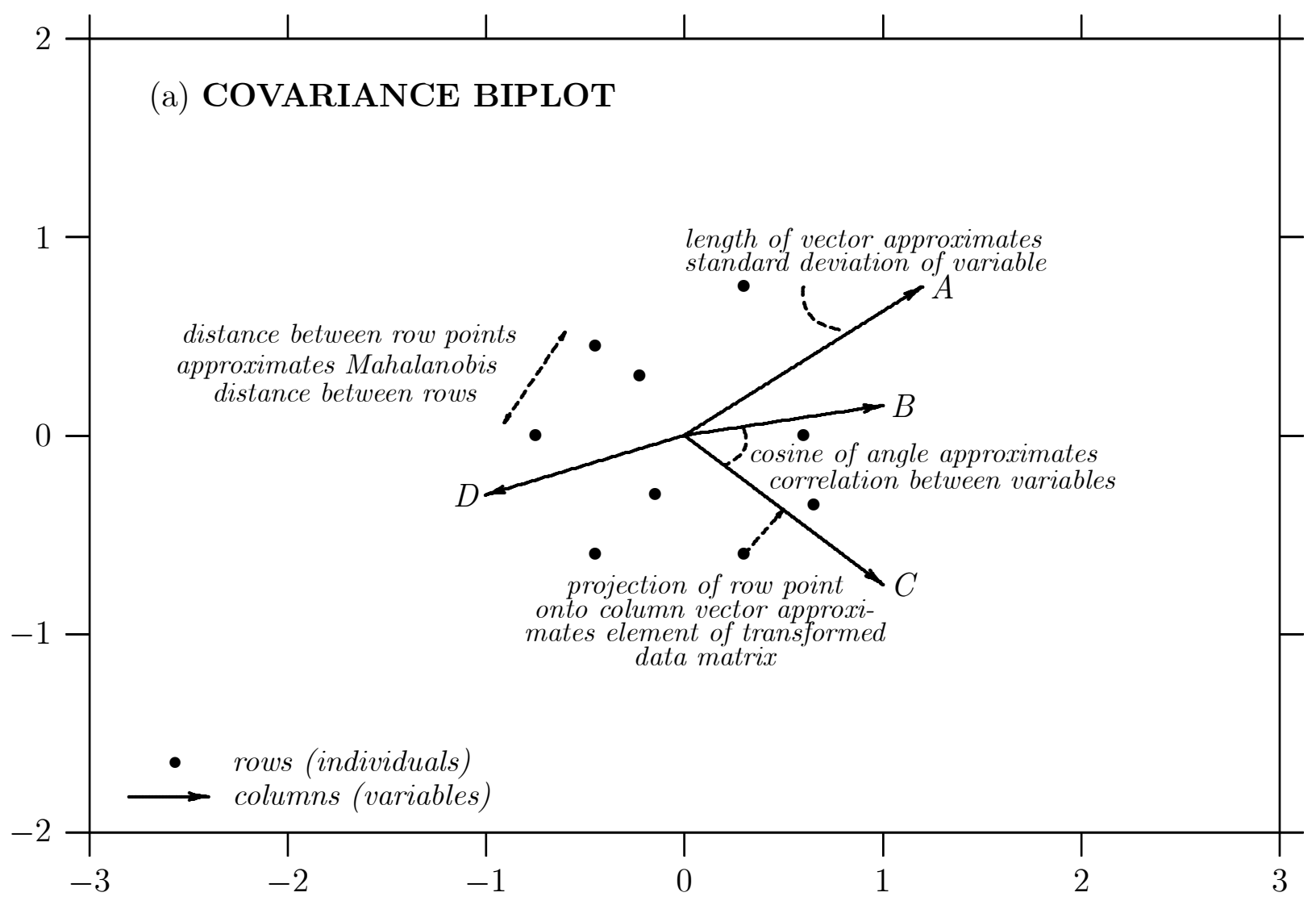




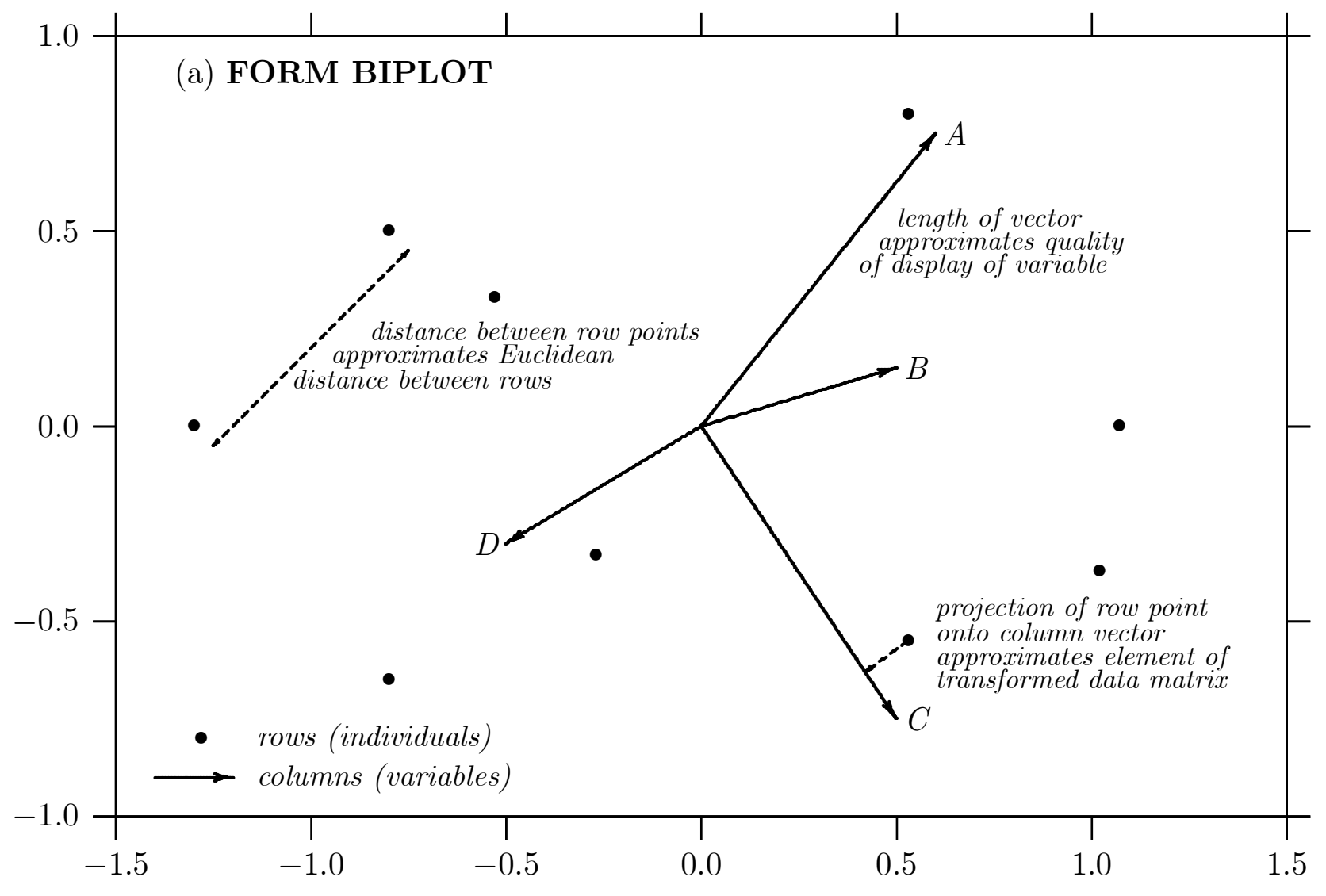


Figure 2

Biplot axes through rays and links.

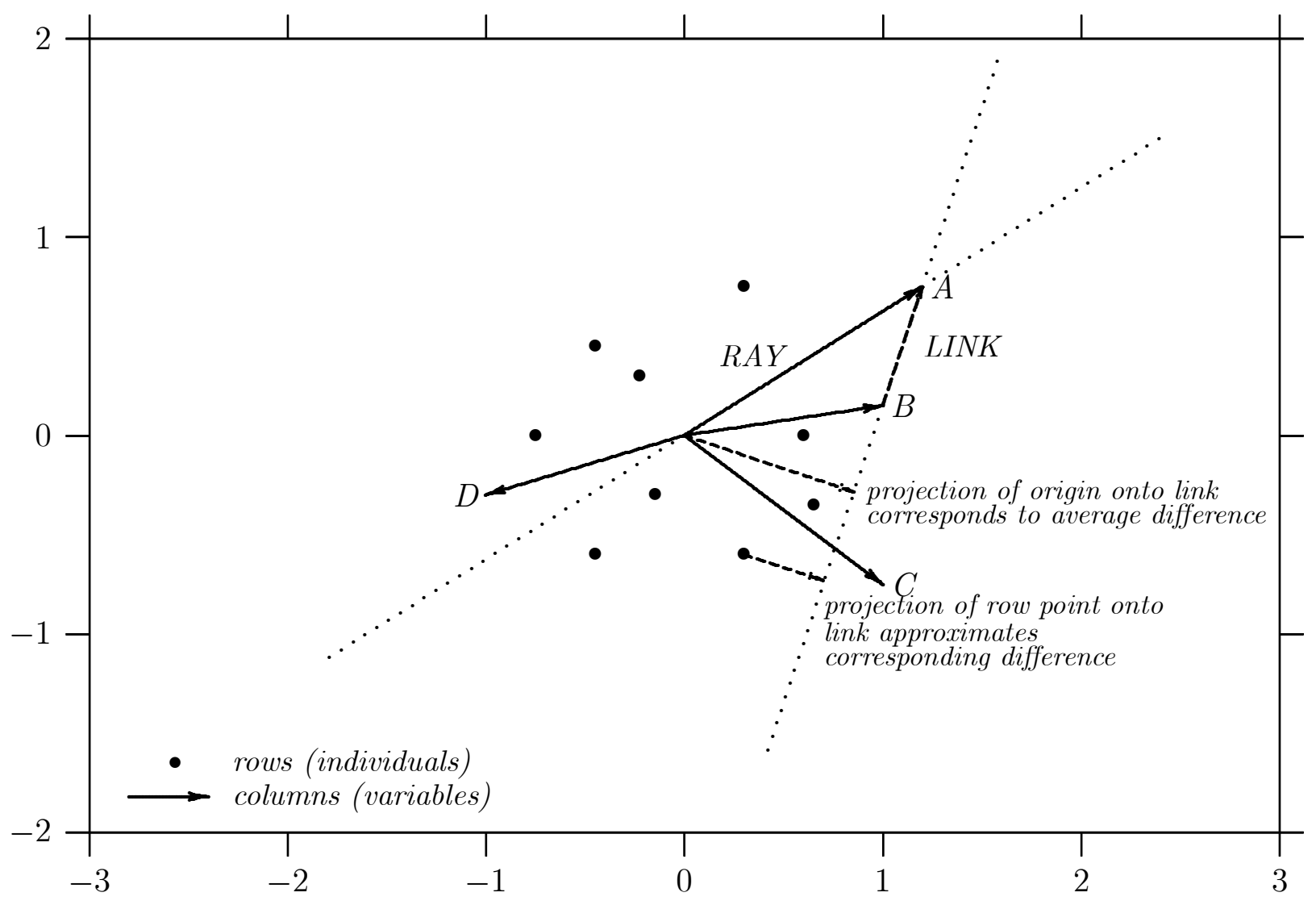




\section{Figure 3}

Relative variation biplot of colour composition data, preserving distances between rows (paintings).

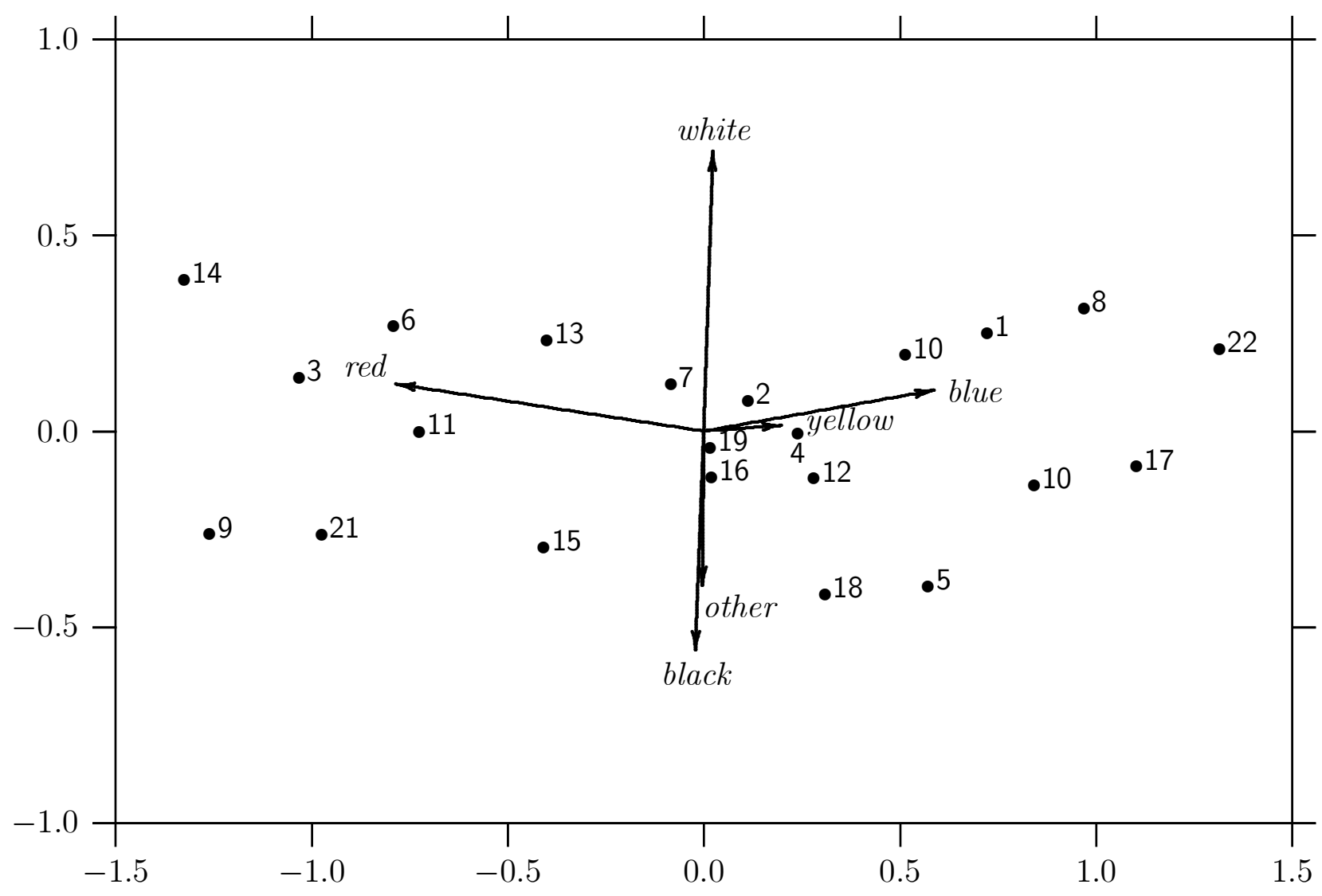




\section{Figure 4}

Relative variation biplot of colour composition data, preserving covariance structure between logratios.

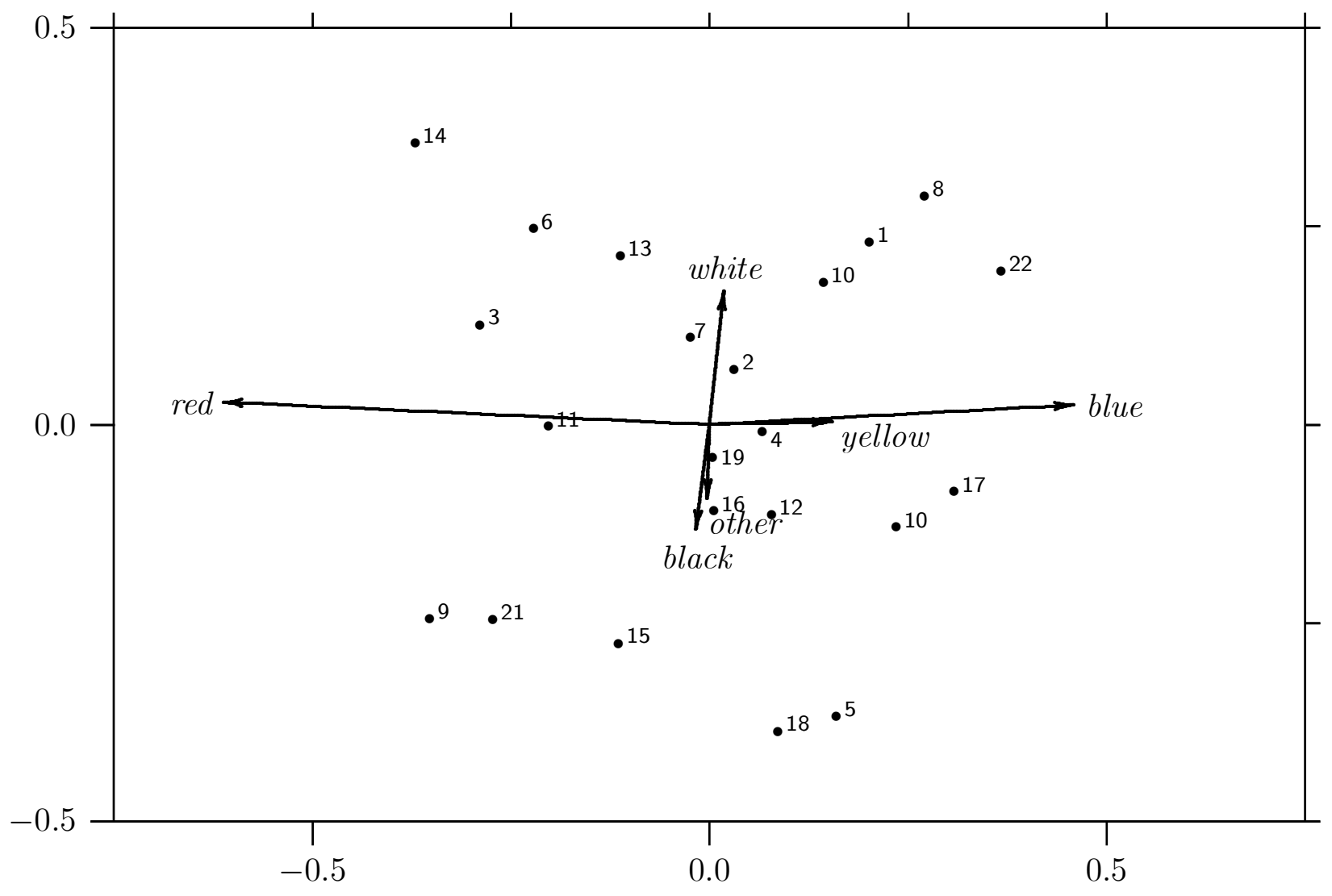


Figure 5

Relationship between colour ratios red/yellow and (yellow/blue) ${ }^{2.5}$, showing proportionality relationship.

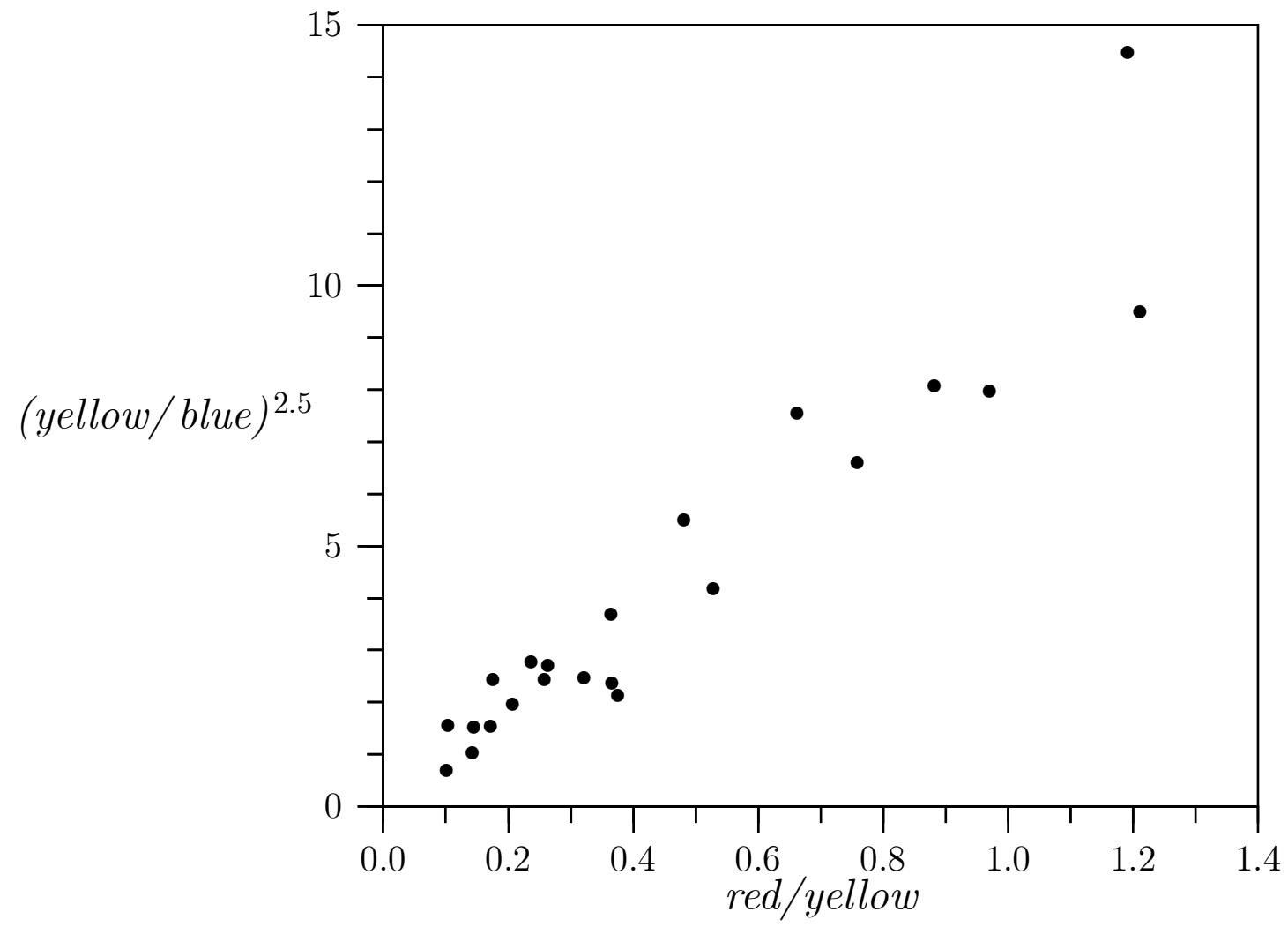


Figure 6

Goethe's colour triangle, showing mixtures of primary colours in 22 paintings, and model diagnosed by the relative variation biplot: $($ red/yellow $) \propto(\text { yellow/blue })^{2.5}$.

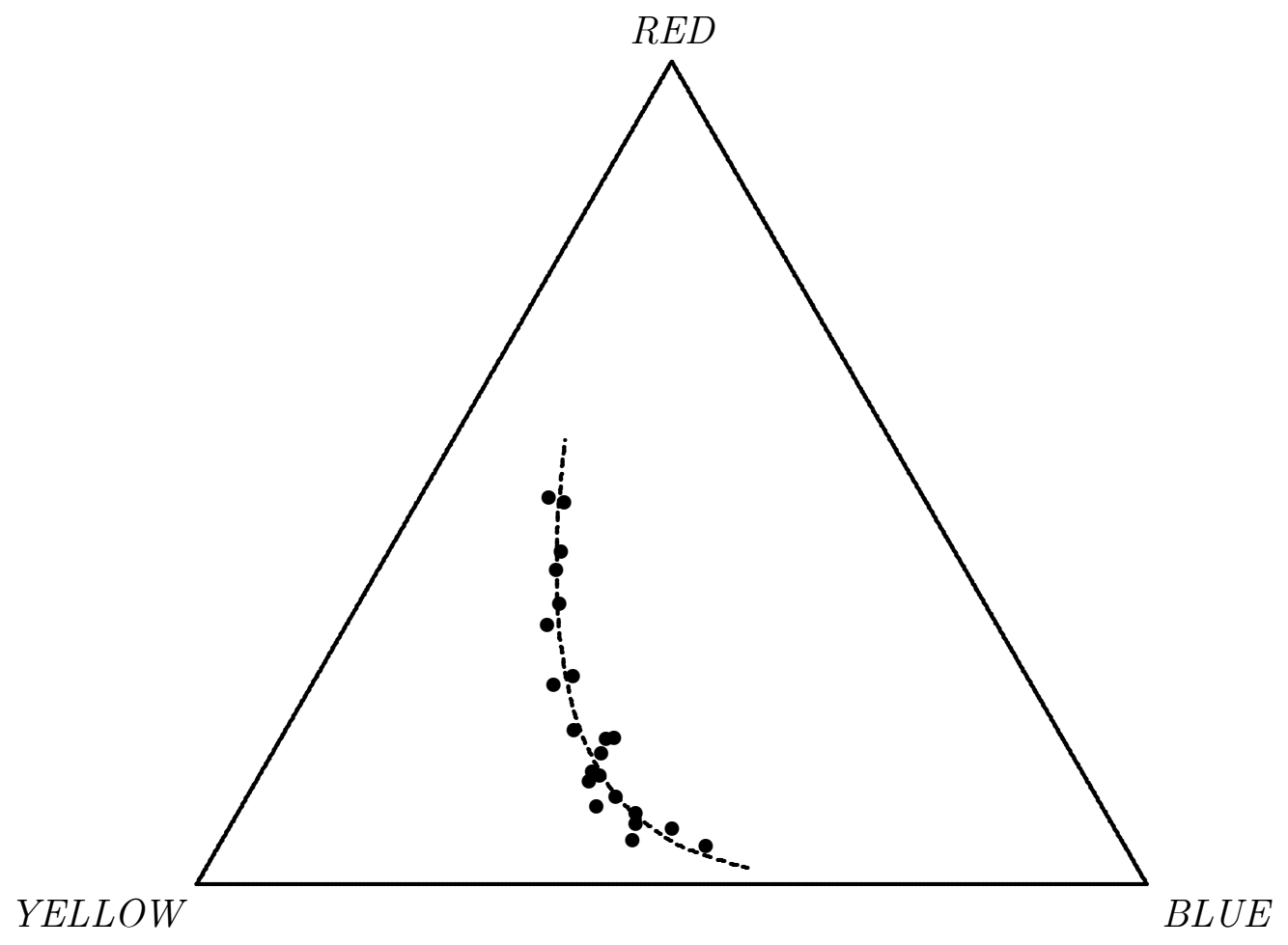




\section{Figure 7}

Relationship between colour ratios black/red and blue/white, showing proportionality relationship.

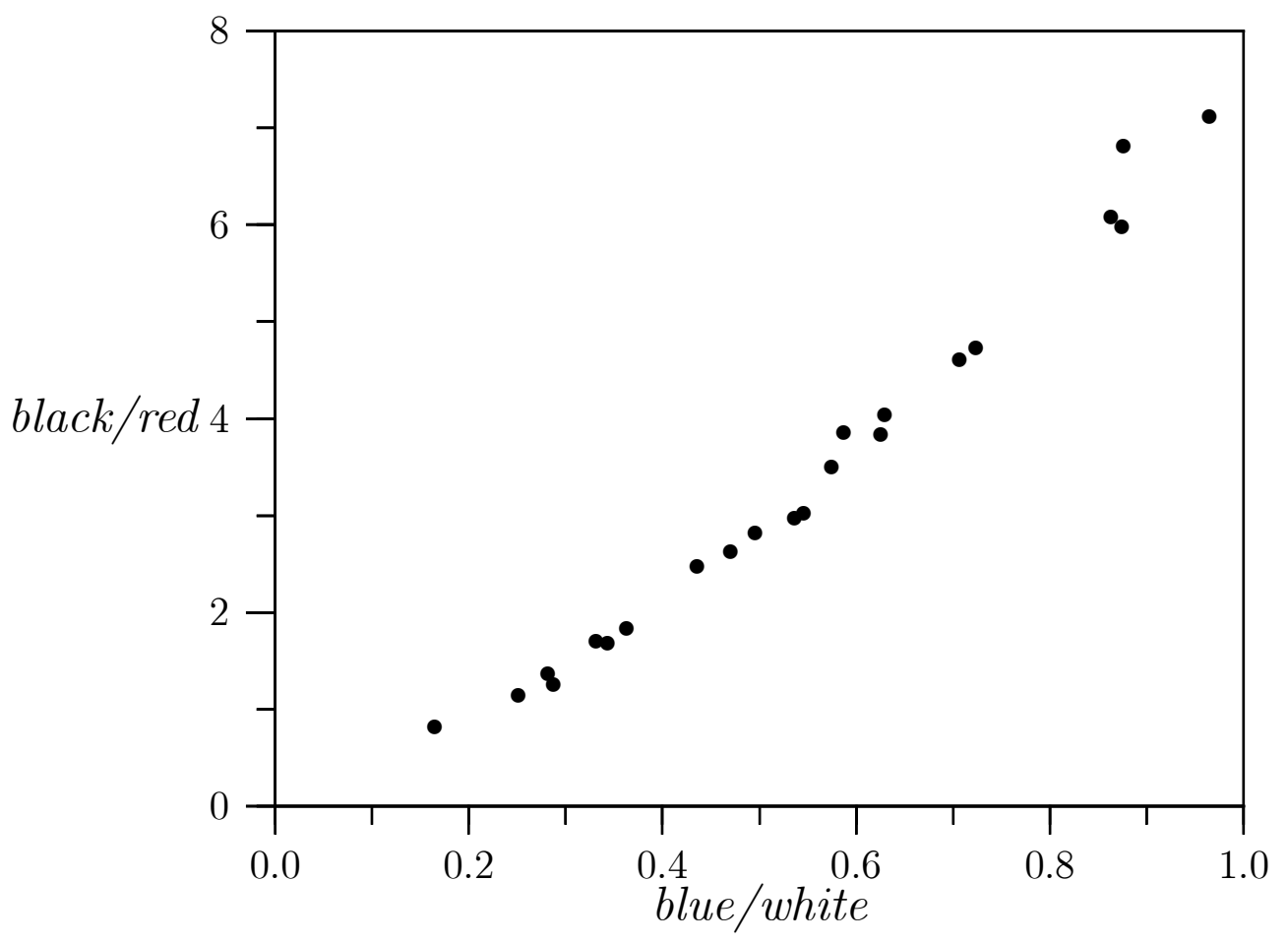




\section{Figure 8}

Logratio covariance biplot of colour composition data, showing linear calibration of logratios $\log ($ yellow/red) and $\log$ (white/black).

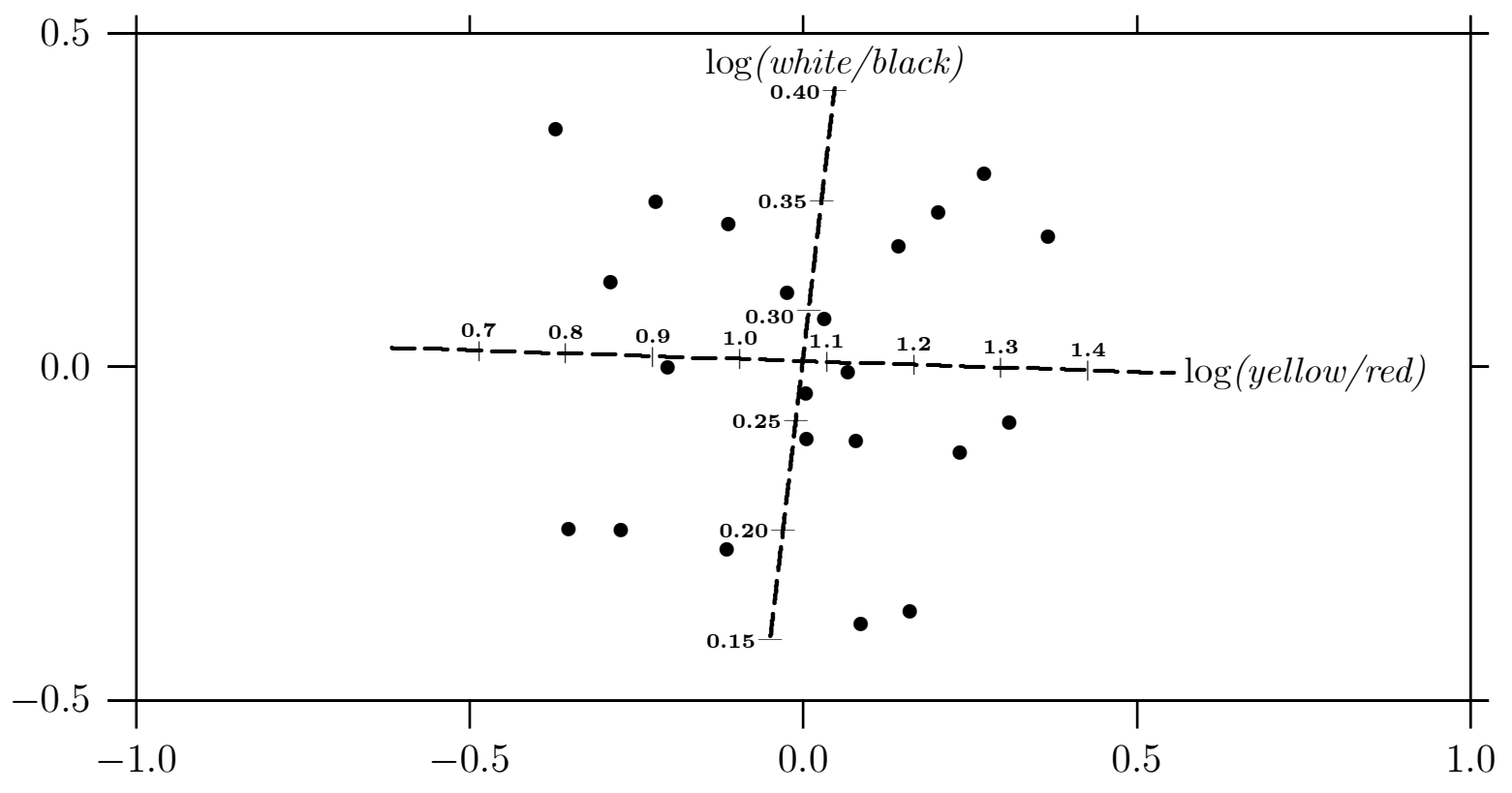




\section{Figure 9}

Logratio covariance biplot of colour composition data, showing nonlinear calibration of ratios yellow/red and white/black on

a logarithmic scale.

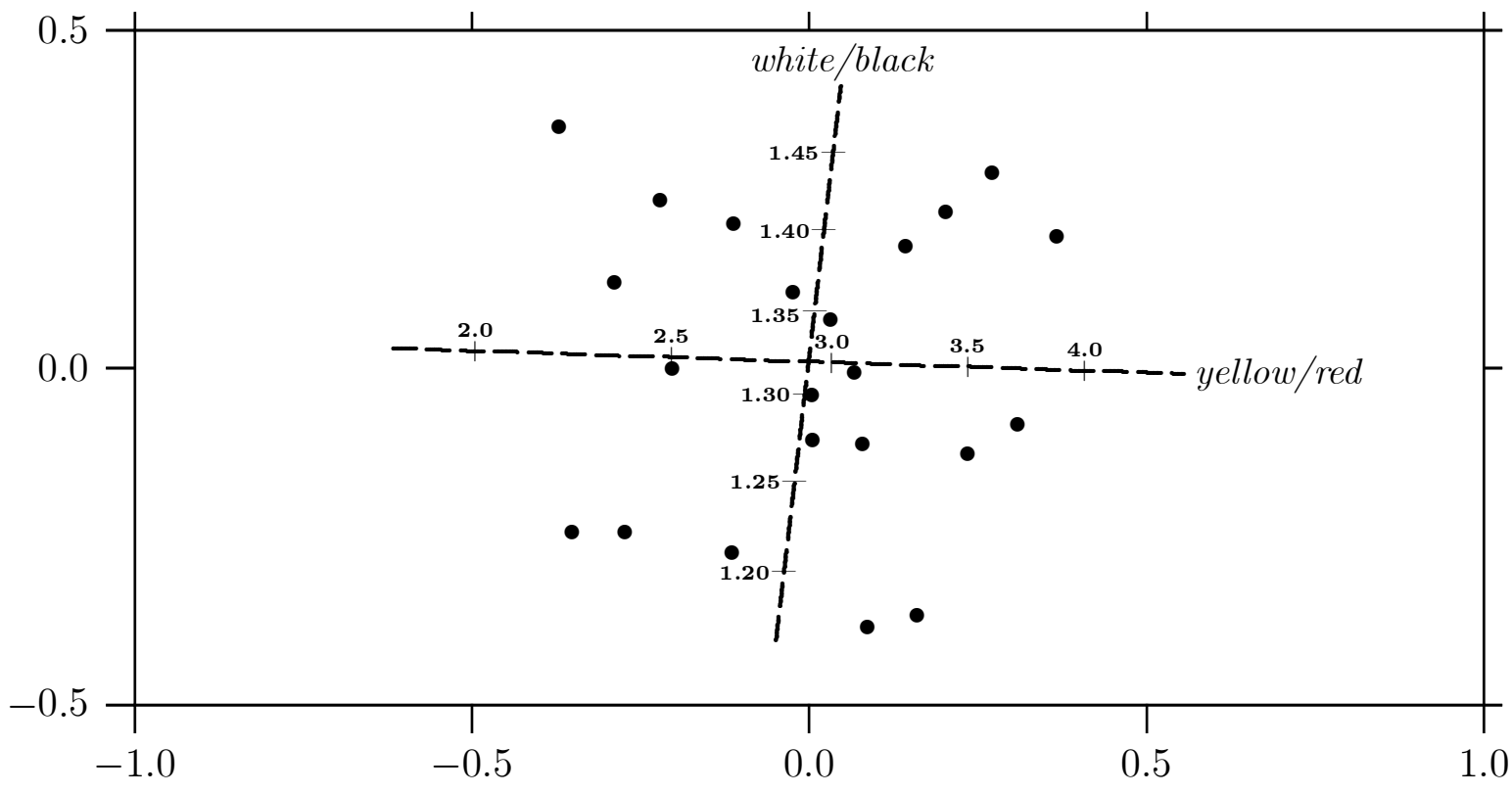


Table 1

Colour composition data for 22 abstract paintings

\begin{tabular}{ccccccc}
\hline Painting & Black & White & Blue & Red & Yellow & Other \\
\hline 1 & 0.125 & 0.243 & 0.153 & 0.031 & 0.181 & 0.266 \\
2 & 0.143 & 0.224 & 0.111 & 0.051 & 0.159 & 0.313 \\
3 & 0.147 & 0.231 & 0.058 & 0.129 & 0.133 & 0.303 \\
4 & 0.164 & 0.209 & 0.120 & 0.047 & 0.178 & 0.282 \\
5 & 0.197 & 0.151 & 0.132 & 0.033 & 0.188 & 0.299 \\
6 & 0.157 & 0.256 & 0.072 & 0.116 & 0.153 & 0.246 \\
7 & 0.153 & 0.232 & 0.101 & 0.062 & 0.170 & 0.282 \\
8 & 0.115 & 0.249 & 0.176 & 0.025 & 0.176 & 0.259 \\
9 & 0.178 & 0.167 & 0.048 & 0.143 & 0.118 & 0.347 \\
10 & 0.164 & 0.183 & 0.158 & 0.027 & 0.186 & 0.281 \\
11 & 0.175 & 0.211 & 0.070 & 0.104 & 0.157 & 0.283 \\
12 & 0.168 & 0.192 & 0.120 & 0.044 & 0.171 & 0.305 \\
13 & 0.155 & 0.251 & 0.091 & 0.085 & 0.161 & 0.257 \\
14 & 0.126 & 0.273 & 0.045 & 0.156 & 0.131 & 0.269 \\
15 & 0.199 & 0.170 & 0.080 & 0.076 & 0.158 & 0.318 \\
16 & 0.163 & 0.196 & 0.107 & 0.054 & 0.144 & 0.335 \\
17 & 0.136 & 0.185 & 0.162 & 0.020 & 0.193 & 0.304 \\
18 & 0.184 & 0.152 & 0.110 & 0.039 & 0.165 & 0.350 \\
19 & 0.169 & 0.207 & 0.111 & 0.057 & 0.156 & 0.300 \\
20 & 0.146 & 0.240 & 0.141 & 0.038 & 0.184 & 0.250 \\
21 & 0.200 & 0.172 & 0.059 & 0.120 & 0.136 & 0.313 \\
22 & 0.135 & 0.225 & 0.217 & 0.019 & 0.187 & 0.217 \\
\hline & & & & & &
\end{tabular}


Table 2

Upper triangle: standard deviations $\sqrt{ } \operatorname{var}\left\{\log \left(x_{i} / x_{j}\right)\right\}$ of logratios.

Lower triangle: estimated values from link lengths in biplot of Figure 4.

\begin{tabular}{lcccccc}
\hline & black & white & blue & red & yellow & other \\
\hline black & $\cdot$ & 0.308 & 0.504 & 0.616 & 0.225 & 0.130 \\
white & 0.302 & $\cdot$ & 0.466 & 0.645 & 0.221 & 0.270 \\
blue & 0.501 & 0.463 & $\cdot$ & 1.071 & 0.315 & 0.488 \\
red & 0.616 & 0.646 & 1.071 & $\cdot$ & 0.767 & 0.628 \\
yellow & 0.218 & 0.214 & 0.305 & 0.767 & $\cdot$ & 0.213 \\
other & 0.041 & 0.262 & 0.476 & 0.621 & 0.184 & $\cdot$ \\
\hline
\end{tabular}


Table 3

Submatrix of correlation matrix between selected logratios amongst two sets of points following perpendicular straight-line patterns in Figure 4, as well as another logratio, $\log ($ yellow/white), for comparison purposes.

\begin{tabular}{lccccccc}
\hline Logratio & $\begin{array}{c}\text { red/ } \\
\text { yellow }\end{array}$ & $\begin{array}{c}\text { red/ } \\
\text { blue }\end{array}$ & $\begin{array}{c}\text { yellow/ } \\
\text { blue }\end{array}$ & $\begin{array}{r}\text { white/ } \\
\text { other }\end{array}$ & $\begin{array}{c}\text { other/ack } \\
\text { black }\end{array}$ & $\begin{array}{c}\text { white/ } \\
\text { black }\end{array}$ & $\begin{array}{c}\text { yellow/ } \\
\text { white }\end{array}$ \\
\hline $\begin{array}{l}\text { red/yellow } \\
\text { red/blue }\end{array}$ & 1.000 & 0.996 & 0.949 & -0.048 & -0.095 & -0.082 & 0.654 \\
yellow/blue & 0.996 & 1.000 & 0.974 & -0.074 & -0.108 & -0.110 & 0.616 \\
& & 0.974 & 1.000 & -0.133 & -0.138 & -0.175 & 0.502 \\
white/other & -0.048 & -0.074 & 0.133 & 1.000 & 0.069 & 0.907 & 0.638 \\
other/black & -0.095 & 0.108 & 0.138 & 0.069 & 1.000 & 0.482 & 0.291 \\
white/black & -0.082 & 0.110 & 0.175 & 0.907 & 0.482 & 1.000 & 0.683 \\
& & & & & & & \\
yellow/white & 0.654 & 0.616 & 0.502 & 0.638 & 0.291 & 0.683 & 1.000 \\
\hline
\end{tabular}


Table 4

Submatrix of colour data, identified by perpendicular straight lines, showing near-constant ratios across paintings

\begin{tabular}{ccccccc}
\hline Painting & Black & White & Other & Black/White & Other/White & Other/Black \\
\hline 9 & 0.178 & 0.167 & 0.347 & 1.07 & 2.08 & 1.95 \\
15 & 0.199 & 0.170 & 0.318 & 1.17 & 1.88 & 1.60 \\
21 & 0.200 & 0.172 & 0.313 & 1.16 & 1.82 & 1.65 \\
\hline
\end{tabular}


Table 5

Reconstructed compositional data from two-dimensional calibrated biplot (cf. original data in Table 1).

\begin{tabular}{ccccccc}
\hline Painting & Black & White & Blue & Red & Yellow & Other \\
\hline 1 & 0.131 & 0.245 & 0.156 & 0.031 & 0.182 & 0.254 \\
2 & 0.154 & 0.225 & 0.113 & 0.052 & 0.170 & 0.287 \\
3 & 0.155 & 0.232 & 0.059 & 0.131 & 0.137 & 0.285 \\
4 & 0.160 & 0.210 & 0.119 & 0.046 & 0.172 & 0.293 \\
5 & 0.187 & 0.152 & 0.132 & 0.032 & 0.173 & 0.324 \\
6 & 0.144 & 0.257 & 0.069 & 0.111 & 0.145 & 0.272 \\
7 & 0.153 & 0.233 & 0.102 & 0.062 & 0.165 & 0.285 \\
8 & 0.122 & 0.250 & 0.176 & 0.025 & 0.186 & 0.240 \\
9 & 0.189 & 0.168 & 0.048 & 0.145 & 0.127 & 0.324 \\
10 & 0.160 & 0.183 & 0.158 & 0.027 & 0.182 & 0.290 \\
11 & 0.167 & 0.212 & 0.070 & 0.102 & 0.146 & 0.302 \\
12 & 0.169 & 0.192 & 0.120 & 0.044 & 0.172 & 0.304 \\
13 & 0.146 & 0.253 & 0.087 & 0.081 & 0.157 & 0.276 \\
14 & 0.133 & 0.269 & 0.050 & 0.167 & 0.128 & 0.254 \\
15 & 0.192 & 0.170 & 0.080 & 0.075 & 0.152 & 0.332 \\
16 & 0.172 & 0.195 & 0.104 & 0.055 & 0.166 & 0.309 \\
17 & 0.150 & 0.185 & 0.180 & 0.021 & 0.187 & 0.277 \\
18 & 0.193 & 0.152 & 0.115 & 0.040 & 0.167 & 0.332 \\
19 & 0.166 & 0.206 & 0.105 & 0.056 & 0.166 & 0.301 \\
20 & 0.139 & 0.239 & 0.140 & 0.038 & 0.179 & 0.265 \\
21 & 0.190 & 0.171 & 0.057 & 0.117 & 0.136 & 0.328 \\
22 & 0.123 & 0.224 & 0.205 & 0.018 & 0.190 & 0.239 \\
\hline
\end{tabular}

